\title{
Mental Health with Stigma \& Nurturing Resilience during COVID-19 Outbreak
}

Dr. Jaya Bharti*

Assistant Professor Department of Psychology A.N.D.N.N.M.M. Harshnagar Kanpur, Kanpur University, India

\author{
DOI: $10.36348 /$ sjnhc.2020.v03i07.001 $\quad$ | Received: 01.07.2020 | Accepted: 08.07.2020 | Published: 15.07 .2020 \\ *Corresponding author: Dr. Jaya Bharti
}

Abstract

Background: The COVID-19 pandemic is a major health crisis affecting India like other countries, with over 42,533 confirmed cases and 1373 deaths till 04 may 2020. This spread outbreaks are associated with adverse mental health consequences caused confusion, changed Quality of Life, including movement restrictions, fear of disease become panic, and closure of schools, shops and businesses, and brought about overwhelming psychological impacts, like anxiety, depression, stress, the psychological impact of COVID-19 and its relation with mental health were examined. Because of stigmatization and the fear of being labelled as someone who carries an infectious disease many at risk populations may not seek care until symptoms are unmanageable or may not seek care at all. Resilience is the capacity to cope up with stress and adversity. It is universally regarded as a positive and valued trait. Purpose of resilience is development of initiative, self-regulation and attachment, which are protective factors that can offset or moderate the effects of stress and adversity and allow to everyone to thrive of transformed by adversity. Specially, in situations of corona virus is important to nurturing resilience in individual, family and community. Methodology: In this short review, accordingly, the electronic databases, including PubMed, Medline, Elsevier, and Science Direct, Embase, PsycINFO, Google scholar, Scopus were reviewed for relevant studies (with key words )that have conducted since 2014. Results: The current review paper was carried out to indicate that COVID-19 has negative psychological problems with situational difficulties like patient isolation; contact tracing, quarantine, lockdown and infection control methods should also address the risk of stigmatization among populations and the negative effects which could occur. A review of the studies performed in other parts of the world showed that COVID-19 has caused several psychological impacts, including increased anxiety. Conclusions: By an increase in the prevalence rate of COVID-19 and its resulted movement restrictions, the level of anxiety increases, as well; therefore, there is the need to public awareness of the disease programmes and providing positive psychological intervention programs for controlling stress and reduce anxiety in general population as well as addressing stigma and discrimination targeted toward individuals affected by COVID-19 and groups at higher risks is a priority for public health and health care providers. Individual resilience is a person's ability to positively cope after failures, setbacks, and losses. Developing resilience is a personal journey. Individuals do not react the same way to traumatic or stressful life events. An approach to building resilience that works for one person might not work for another.

Keywords: Mental Health Nurturing Resilience Covid-19 Outbreak.

Copyright @ 2020: This is an open-access article distributed under the terms of the Creative Commons Attribution license which permits unrestricted use, distribution, and reproduction in any medium for non-commercial use (NonCommercial, or CC-BY-NC) provided the original author and source are credited.

\section{INTRODUCTION}

The COVID-19 is regarded as one of the biggest global health crisis in recent decades, and billions of people around the world are preoccupied by concerns over such a pandemic. The lives of many people, not only those who got infected and their families, but also the general public, have been substantially affected by the pandemic. Fear and anxiety have sparked along with the increasing number of diagnosed cases, and stigma and discrimination arise towards Indians, as well as people who have contact with those infected patients. As social workers who cherish the value of human dignity and respect, and strive for social justice, we need to realise that the real danger is not only the virus itself, but also the social panic, stigma and discrimination provoked by this pandemic. And we social workers need to take action.

Pandemics and epidemics of new and reemerging infectious disease are unpredictable but recurrent events that threaten global health and socioeconomic security. It is of utmost importance to human health that there is capacity to conduct time- critical, 
patient- centred research during a pandemic because such research offers the best strategy for mitigating impact on health and society [1-3]. Such research is necessary to help clinicians accurately diagnose and provide optimal treatment, to develop preventive interventions such as vaccines \& policymakers to make decisions about strategies to prevent spread and for mobilize mental health- care services for general population.

Li \& Guan [4] stated that since the end of December 2019, the Chinese city ofWuhan has reported a novel pneumonia caused by coronavirus disease 2019 (COVID-19), which is spreading domestically and internationally. The virus has been named severe acute respiratory syndrome coronavirus 2 (SARS-CoV-2) [5]. On January 30, 2020, World Health Organization held an emergency meeting and declared the global COVID19 outbreak a public health emergency of international concern [6].

In this pandemic situation, Administration, shoppers, Municipality employees, Doctors, nurses, attendants, ambulance drivers and Social workers who are directly involved in the food supply, water supply, cleaning, manage daily activites, diagnosis, treatment, and care of patients with COVID-19, more at risk of developing psychological distress, anxiety and depression. The ever-increasing number of confirmed and suspected cases, overwhelming workload, depletion of personal protection equipment, widespread media coverage, lack of specific drugs, and feelings of being inadequately supported may all contribute to the mental burden of these health care workers [7-10].

Family members of front line staff who are involved in the management of COVID-19, students, elderly, middle age women's, caregivers of ill patients reported experiencing high levels of stress, anxiety, and depression symptoms, which could have long-term psychological implications. With the above objectives in mind, the current review was designed to summarize the existing literature addressing mental health concerns related to the COVID-19 pandemic. A recent study involving 1,563 health professionals found that more than half $(50.7 \%)$ of the participants reported depressive symptoms, $44.7 \%$ anxiety, and $36.1 \%$ sleep disturbance [11]. According to China News Weekly [12] Patients with severe mental illness (SMI) are inevitably affected by the COVID-19 outbreak. In early February, 2020, over 40 inpatients in Wuhan Mental Health Center were diagnosed with the COVID-19.

"Resilience is accepting your new reality, even if it's less good than the one you had before. You can fight it ... or you can accept that and try to put together something that's good."

The crisis is an opportunity for us to have a straightforward discussion about our need to make our families, communities, workplaces, and government more resilient. Thinking about the many different systems we need for mental and physical health, from a positive attitude to social network and health care, opens up positive attitude to social networks and health care, opens up possibilities to make as much as more proficient of withstanding long periods of forced isolation, financial stress, gloominess, distemper and mourning.

\section{During this corona virus, we need to}

- Maintain structure in our lives, getting up in the morning at the regular time and keeping as many routines as possible.

- Find ways to focusing on our roles as a parent or helping to look after someone else's pet if they leave their home. The more accountable and easier it is to maintain our mental health.

- Reach out continue to nurture our social connections, whether online (using what's app, Facebook and other social networking site), or by checking in on friends and family.

- Opportunity to strengthen different parts of our identity, whether that is taking up a new hobby and other different showing side of our personality.

- Take care of our health. Stay active always and prevent health problem that are pandemic.

- Resilience is able to effectively cope with recover from or adapt to challenging life situations. A person who is able to cope with crisis situations due to behaviours, thoughts and actions that they have learned and developed. There are some common factors include having:

\section{METHODOLOGY}

The current article is a narrative review of the existing literature on mental health symptoms and interventions relevant to the COVID-19 pandemic for nurturing resilience. In this short review, accordingly, the electronic databases, including PubMed, Medline, Elsevier, and Science Direct, Embase, PsycINFO, Google scholar, Scopus were reviewed for relevant studies conducted since 2014, using the search terms novel coronavirus, COVID-19, mental health, mental health care professionals, Ebola, anxiety, depression and stress in various combinations.

\section{RESULT}

The outbreak of the COVID-19 has caused tremendous psychological problems in every person of the world. On the basis of these recent experiences, the National Health Commission of China released a notification on January 26, 2020, providing guiding principles of the emergency psychological crisis interventions to reduce the psychosocial effects of the COVID-19 outbreak. Al-Rabiaah [13] explored the impacts of the Middle East respiratory syndrome coronavirus (MERS-CoV) epidemic by examining medical students and found that all of these students 
experienced stress; however, female students were found with higher levels of stress. Van Bortel et al. [14] assessed the psychological impacts of the Ebola outbreak on people, society, and the world. This disease infected nearly 28,000 people from 2013 to 2016 and led to 11,000 deaths. The results demonstrated that people experienced severe psychological trauma due to observing other people's death and having a fear of death. Lee et al. [9] evaluated the psychological impacts of the MERS outbreak and found that PTSD symptoms were very high among hospital staff even many years after the outbreak. Widespread outbreaks of infectious disease, such as COVID-19, are associated with psychological distress and symptoms of mental illness [15]. Psychiatrists across the world should be aware of these manifestations, their correlates, and strategies to manage them that encompass both the needs of specific populations [16] and the precautionary measures necessary to contain the spread of COVID-19 [4]. They should also be aware of lacunae in the existing literature, which may need to be filled in over time through more widespread clinical experience and research. Fear, denial and frustration, which comprise three sequential stages of the rational response to fear, have been reported as predominant among patients or quarantined individuals during the COVID-19 outbreak in all over the world. That's showed poor psychological wellbeing in general population as we know that people with lower psychological wellbeing are also more susceptible to various inflammations in the body and to immune system disorders and also stated by the doctors that Covid-19 disease is more common in people with low immune system or Covid-19 disease affects people with weaker immune system.

A study conducted in Iran by Zandifar and Badrfam [17] connoting the role of unpredictability, uncertainty, seriousness of the disease, misinformation and social isolation in contributing to stress and mental morbidity. The authors highlighted the need for both mental health services, particularly for vulnerable populations, and the strengthening of social capital to reduce the adverse psychological impact of the outbreak. In addition, psychiatric disorders, such as depression, anxiety, and posttraumatic stress disorder, developed in high-risk persons, especially survivors and frontline healthcare workers [18]. COVID-19 creates heightened anxiety and poorer psychological wellbeing in human beings. Heightened anxiety is significantly associated with increased perception of susceptibility to infection and social avoidance behaviours related to travel and being in public places: mall, theatre, shops, neighbour etc.

\section{Boost our resilience levels in a number of ways, including the following:}

1. Keep a positive outlook - Having an optimistic attitude can help you focus on the good things in your life, rather than worrying about things you may not be able to control. In this situation you need to acknowledge your own strength, resourcefulness and abilities. This will help you avoid seeing the pandemic as an insurmountable crisis. For example: If you have children may feel anxious or fearful about their families or their own personal health so, you need to create a positive atmosphere in these difficult situations.

2. Develop realistic goals and decisive action - if you have a plan so, focus on specific tasks that will enable you to move towards your goal. This might include ensuring you have a supply of food ,water, flu- related medicine or activities that you and your family can do at home if you become isolated .By keeping yourself busy, your mind will be occupied ,having less time to dwell on worries and fears.

3. Control stress by managing your worries - Intense worrying about what might happen or how the situation with ill family members might get worse can trigger ours body automatic Fight-flight stress response. Overtime, this physical response to stress takes its toll on our bodies. Consider finding effective ways of managing stress reason of quarantine the worry it is create a regular half hour each day as worry time to identify and tackle each worry as if were a problem to solve. When you feel yourself slipping into a worried frame of mind, try to postpone the feeling and focus instead on what is actually happening at that moment.

4. Past learning - To think about past experiences and sources of personal strength that helped you through other crisis situation. For example: How did you overcome the difficult situations?

Since a pandemic can last a number of weeks, need to consider about stocking up on non-perishables foods, water, medicine and necessary grocery, etc. Stocking up is necessary if officials recommend staying home or you're self-isolate due to a family member becoming ill. And also developing a plan for how you would care for sick family members. You can also create an emergency communication plan to keep in touch with other family members or friends if you or they become isolated and use community resources or websites to create a family preparedness during pandemic situations.

\section{Nurturing Resilience as a virtue}

According to Russell's definition, "resilience is a virtue Aristotle acknowledges that virtues are not just some traits of individuals. They are complex states that are expressed in habitual actions over time and in the right contexts. They are nurtured through experience, encouragement, social supports, opportunities, and choose, plan and act wisely in adapting to adversity". Resilience is a number of ways, as well as used in a variety of contexts. In the biological sciences, resilience is described as an ability to make an adjustment in behaviour toward adaptability [19]. Resilience in ecological contexts is the capacity of a system to absorb and adapt to unforeseen future events [20]. 
Psychological resilience, which is the human capacity to cope with stress and adversity [21]. It is described as the ability to bounce back from difficult times and human capacity to overcome challenging stressors and become competent, confident and caring individuals [22]. There is the positive, adaptive response in the face of significant adversity [23].

Resilience is interconnected with other virtues such as courage, temperance, patience, imaginations, willingness to be self-critical, and wisdom. Together this virtual support a mean between two states of deficiency and excess, each is representing a vice. For examples: A courageous person who does not have patience may be led to foolhardy exploits. An individual who shows little or no resilience may lack of courage

On the other hand, courage without a willingness to be self- critical may blind the person to a needed area of adaptation. The resilient person needs experience and learning to hit the mean which involves nurturing the other virtues. Resilience is not only dynamic, but also contextual and malleable. It is not type a trait. While there are personality dimensions that may be associated with resilience, it is recognized that experiences shape personality trait, which in turn can influence, depending on the domain of adaptation, the physical or socio-cultural value and meaning of the trait, age and gender of the individual [24]. However, protective mechanisms that have been remarkably consistent in the literature across the time that seems to give rise to successful.

\section{Individual/Personal or Family Resilience}

A. Individual resilience is a person's ability to positively cope after failures, setbacks, and losses.

B. Developing resilience is a personal journey. Individuals do not react the same way to traumatic or stressful life events. An approach to building resilience that works for one person might not work for another.

C. People use varying strategies to build their resilience. Because resilience can be learned, it can be strengthened.

D. Personal resilience is related to many factors including individual health and wellbeing, individual aspects, life history and experience, and social support.

E. Family resilience is the coping process in the family as a functional unit.

F. Crisis events and persistent stressors affect the whole family, posing risks not only for individual dysfunction, but also for relational conflict and family breakdown.
Following are the three key factors in family resilience [25].

a) By making meaning in adversity, creating a sense of coherence, and providing a positive outlook.

b) By facilitating flexibility, capacity to adapt, connectedness and cohesion, emotional and structural bonding, and accessibility to resources.

c) Family communication enhances resilience by engaging clear communication, open and emotional expressions, trust and collaborative problem solving, and conflict management.

\section{Community or Organizational Resilience}

- It is a community that takes intentional action to enhance the personal and collective capacity of its citizens and institutions to respond to and influence the course of social and economic change.

- For a community to be resilient, its members must put into practice early and effective actions so that they can respond to change.

- When responding to stressful events, a resilient community will be able to strengthen community bonds, resources, and the capacity to cope.

Organizational resilience is the ability and capacity of a workplace to withstand potential significant economic times, systemic risk, or systemic disruptions by adapting, recovering, or resisting being affected and resuming core operations or continuing to provide an acceptable level of functioning and structure.

- The challenge for the incorporation of resilience into a workplace is to identify what enhances the ability of an organization to rebound effectively.

Measuring workplace resilience involves identifying and evaluating the following

- Past and present mechanisms and practices that increase safety.

- Past and present mechanisms and practices that decrease error.

- Necessary redundancy in systems.

- Planning and programming that demonstrate collective mindfulness.

- Anticipation of potential trouble and solutions to potential problems.

\section{Factors contributing to community resilience}

A. Human capital - It is innate and acquired personal attributes including skills and knowledge.

B. Social capital - Social network and connections within a community, effectiveness of community and organisations.

C. Build community resilience 
D. Strong social network and availability of resources are explanation to community recovery of adversity. Interventions which focus to mobilise and maintain and improve community resources are essential where the existing social support. Focus of strengthening resilience response during pandemic:

a) Enhancing social capital

b) Supporting community competence and capacity

c) Strengthening provision and spreading information

\section{Fostering community resilience}

Fostering community resilience will greatly depend on the community itself and involves the community working as a whole.

\section{Community resilience involves the following factors}

A. Connection and caring

B. Collective resources

C. Critical analysis of the community

D. Skill building for community members, Prevention, preparedness, and response to stressful events.

\section{Culture Influence on Resilience}

A. Refers to a culture's capacity to maintain and develop cultural identity and critical cultural knowledge and practices.

B. An individual's culture will have an impact on how the person communicates feelings and copes with adversity.

C. Cultural parameters are often embedded deep in an individual.

D. A person's cultural background may influence one deeply in how one responds to different stressors.

E. Assimilation could be a factor in cultural resilience, as it could be a positive way for a person to manage his/her environment. However, assimilation could create conflict between generations, so it could be seen as positive or negative depending on the individual and culture. Because of this, coping strategies are going to be different.

\section{CONCLUSIONS}

In addition to endangering human health and resulting death, COVID-19 has very bad psychological effects on human societies like complete quarantine lockdown, full lockdown and movement restrictions, which prevent people from going out, fear of suffering from illness, worry of losing loved ones, and more importantly, Depression and facing PTSD (Post Traumatic Stress Disorder) after losing family and relatives are some of the issues that people should deal with own. Currently, at the high peak of the COVID-19 outbreak, there is no detailed information on the psychological impacts of the disease on people, and people experience severe negative emotions due to the closure of schools and businesses. It has indicated that the prevalence of psychological harm caused by the spread of communicable diseases in society is very considerable. People's fear and anxiety can be increased due to the loss of trust in mental health services. This paper also identified populations at higher risk of adverse mental health outcomes, including patients with COVID-19 and their families, individuals with existing physical or psychiatric morbidity, and healthcare workers. Countries should also consider requesting support and guidance from global mental healthcare authorities and research communities through international collaborations. Although this study limits generalization, it seems that students were able to articulate their views of resilience, coping and strengths clearly and that these perceptions were generally in keeping with research findings resilience among community and individual is critical, As their own resistance will contribute to the adequate and appropriate facilitation of resilience among the youth with whom they work. Their responses not only pointed to need for focus on the risk and resilience, but emphasized that this focus should also be contextually bound. People also emphasis that comes social service and nurture resilience and coping with themselves especially during COVID 19. And they believe that this is achieved through critical self- reflection, selfawareness and commitment to self-care. Resilience building either Individual or Community can begin at any time. Following is information regarding applicable ways to implement resilience practices, as well as situations that could inhibit resilience, situations that enhance resilience, and people who help facilitate the growth of resilience.

\section{REFERENCES}

1. Commission on a Global Health Risk Framework for the Future. (2016). The neglected dimension of global security: a framework to counter infectious disease crises. National Academies Press (US).

2. Lurie, N., Manolio, T., Patterson, A. P., Collins, F., \& Frieden, T. (2013). Research as a part of public health emergency response.

3. Rojek, A. M., \& Horby, P. W. (2016). Modernising epidemic science: enabling patient-centred research during epidemics. BMC medicine, 14(1), 212.

4. Liu, S., Yang, L., Zhang, C., Xiang, Y. T., Liu, Z., Hu, S., \& Zhang, B. (2020). Online mental health services in China during the COVID-19 outbreak. The Lancet Psychiatry, 7(4), e17-e18.

5. National Health Commission of China. (2020). Principles of the emergency psychological crisis interventions for the new coronavirus pneumonia.

6. World Health Organization. (2005). Statement on the second meeting of the International Health Regulations

7. Maunder, R., Hunter, J., Vincent, L. (2003). The immediate psychological and occupational impact of the 2003 SARS outbreak in a teaching hospital. CMAJ, 168(10):1245-1251.

8. Bai, Y., Lin, C.C., Lin, C.Y., Chen, J.Y., Chue, C.M., Chou, P. (2004). Survey of stress reactions among health care workers involved with the SARS outbreak. Psychiatr Serv, 55(9):1055-1057 
9. Lee, A.M., Wong, J.G., McAlonan, G.M. (2007). Stress and psychological distress among SARS survivors 1 year after the outbreak. Can $J$ Psychiatry, 52(4):233-240.

10. Chua, S.E., Cheung, V., Cheung, C. (2004). Psychological effects of the SARS outbreak in Hong Kong on high-risk health care workers. Can J Psychiatry; 49(6):391-393.

11. Liu, Y., Li, J., Feng, Y., (2020b). Critical care response to a hospital outbreak of the 2019- nCoV infection in Shenzhen. China. Crit. Care, 24(1), 56.

12. China News Weekly. About 80 doctors and patients at the Wuhan Mental Health Center were diagnosed with novel coronavirus pneumonia.

13. Al-Rabiaah, A., Temsah, M.H., Al-Eyadhy, A.A., Hasan, G.M., Al-Zamil, F., Al-Subaie, S. (2020). Middle East respiratory syndrome-corona virus (MERS-CoV) associated stress among medical students at a university teaching hospital in Saudi Arabia. J Infect Public Health.

14. Van, Bortel, T., Basnayake, A., Wurie, F., Jambai, M., Koroma, A.S., Muana, A.T. (2016). Psychosocial effects of an Ebola outbreak at individual, community and international levels. Bull World Health Organ, 94(3):210- 4.

15. Bao, Y., Sun, Y., Meng, S., Shi, J., Lu, L. (2020). 2019-nCoV epidemic: address mental health care to empower society. Lancet, 22(395), e37-e38.

16. Yang, Y., Li, W., Zhang, Q., Zhang, L., Cheung, T., Xiang, Y.-T. (2020). Mental health services for older adults in China during the COVID-19 outbreak. Lancet Psychiatry, 7(4), e19.
17. Zandifar, A., Badrfam, R. (2020). Iranian mental health during the COVID-19 epidemic. Asian J. Psychiatr, 51, 101990.

18. Mak, I.W., Chu, C.M., Pan, P.C., Yiu, M.G., Chan, V.L. (2009). Long-term psychiatric morbidities among SARS survivors. Gen Hosp Psychiatry, 31:318-26.

19. Hanson, D.R\& Gottesman, I.I.(2012). Biologically flavoured perspectiveson Garmezian resilience. Development and Psychopathology, 24(2), 363 369.

20. Holling, C. (1973). Resilience and stability of ecological systems. Annual review of ecology and systematics, 4(1), 1-23.

21. Masten, A. S. (2001).Ordinary magic: Resilience processes in development. American psychologist, 56(3), 227.

22. Benard, B. (2004).Resiliency: What we have learned.San Francisco,CA: West ED.

23. Luthar, S., Cicchetti, D., \& Becker, B. (2000). The construct of resilience: A critical evaluation and guidelines for future work. Child Development, 71,543-562.

24. Masten, A. S. (2014). Global perspectives on resilience in children and youth. Child development, 85(1), 6-20.

25. Wilson, S. M., \& Ferch, S. R. (2005). Enhancing resilience in the workplace through the practice of caring relationships. Organization Development Journal, 23(4), 45. 